\title{
Novel insights into the relationship between nonalcoholic fatty liver disease and osteoporosis
}

\author{
Rafał Filip ${ }^{1,2}$ \\ Radosław P Radzki ${ }^{3}$ \\ Marek Bieńko ${ }^{3}$ \\ 'Department of Gastroenterology \\ with IBD Unit, Clinical \\ Hospital 2, Rzeszów, Poland; \\ 'University of Rzeszów, Rzeszów, \\ Poland; ${ }^{3}$ Department of Animal \\ Physiology, University of Life Sciences, \\ Lublin, Poland
}

\begin{abstract}
Excess fat deposition and insulin resistance are considered the main risk factors for nonalcoholic fatty liver disease (NAFLD), and therefore, not surprisingly, the global prevalence of NAFLD increases in parallel with both obesity and type 2 diabetes. Although deterioration of bone homeostasis in patients with NAFLD is commonly observed, its etiology has not been fully elucidated yet. It was shown in several studies that bone tissue seems to be independently associated with NAFLD. A mechanistic perspective puts the liver at the center of mutual interdependencies obviously involving adipose tissue and muscles and also the bone matrix and bone cells, which are relatively novel. In this review, various pathophysiological mechanisms and possible mediating molecules that may interplay between NAFLD and bone tissue are described. Chronic inflammation, vitamin D3, growth hormone, insulin-like growth factor 1, osteopontin, fetuin-A, irisin, osteocalcin, and osteoprotegerin from osteoblasts have been proposed as mediators of mutual interactions among the skeleton, fatty tissue, and liver. Although to date there are still many issues that have not been elucidated, growing evidence suggests that screening and surveillance of bone mineral density in patients with NAFLD should be considered in future strategies and guidelines for NAFLD management.
\end{abstract}

Keywords: nonalcoholic fatty liver disease, nonalcoholic steatohepatitis, inflammation, osteoporosis, bone metabolism

\section{Introduction}

Osteoporosis is becoming a public health problem all over the world. Disability resulting from low-energy fractures, eg, hip or vertebral fractures, is the major concern for early detection and treatment. It is estimated that osteoporosis affects 200 million women worldwide - approximately one-tenth of women aged 60 years, one-fifth of women aged 70 years, two-fifths of women aged 80 years, and two-thirds of women aged 90 years. ${ }^{1}$ Over the past two decades, the level of knowledge has significantly increased; however, most of the osteoporosis cases are underdiagnosed and undertreated in highly as well as in poorly developed countries. It is expected that more than $50 \%$ of all osteoporotic hip fractures will occur in Asia by the year 2050. 2,3

The commonly accepted definition of osteoporosis is: "A systemic skeletal disease characterized by low bone mass and microarchitectural deterioration of bone tissue with a consequent increase in bone fragility and susceptibility to fracture." This definition refers to reduction in bone strength generally due to changes in bone macro- and microstructure accompanied also by decreasing bone mineral density (BMD). ${ }^{1}$ Both conditions in parallel lead to an increased risk of low-energy fractures. Diagnosis is based on dual-energy X-ray absorptiometry (DEXA) with the supportive assessment of bone metabolism marker levels. Nonmodifiable risk factors include age, height, weight, body mass index (BMI), and menopause. The preventable risk factors include
Correspondence: Rafał Filip Department of Gastroenterology with IBD Unit, Clinical Hospital 2, Lwowska 60, Rzeszow, 35-30I, Poland Email r.s.filip@wp.pl

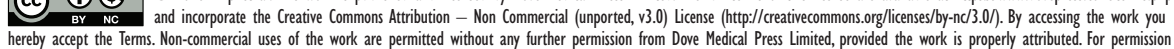
for commercial use of this work, please see paragraphs 4.2 and 5 of our Terms (https://www.dovepress.com/terms.php). 
calcium intake, exposure to sunlight, smoking habit, alcohol intake, level of physical activity, underlying disease conditions such as endocrine or autoimmune disorders, intake of steroids, and other drug therapies among others. ${ }^{3}$

Although osteoporosis is considered to affect postmenopausal women and elderly, it may occur at any time during the course of various gastrointestinal (GI) diseases. ${ }^{4,5}$ Most important pathomechanisms are linked to maldigestion and malabsorption, inflammation, and possibly as a result of various pharmacological therapies. On the contrary, it is common, particularly in those with celiac disease, that osteoporosis may be the only noticeable symptom.

The population of people with GI diseases is increasing, and their diagnosis and treatment impact the general practitioner and specialist health care costs. One important aspect of their physical health is the bone health as people with GI diseases have, apart from those mentioned above, additional risk factors associated with their primary disease. It has been identified that this population has an increased prevalence of low BMD, osteoporosis, and osteopenia. ${ }^{4}$ For example, fracture risk for patients with inflammatory bowel diseases (IBDs) is increased by approximately $40 \%$ to $60 \%$, and in this particular case, the main risk factors for osteoporosis and osteopenia in IBD patients include activity and severity of gut inflammation, perianal disease including fistulae as well as prolonged systemic steroid usage, and malabsorption of selected nutrients. ${ }^{5-7}$ However, it must be pointed out that apart from those with IBD or celiac disease, in patients with GI diseases, the prevalence of bone metabolic diseases was not profoundly studied up to date. ${ }^{4}$ Especially, when considering chronic liver diseases, where possible mechanisms, apart from malnutrition, also include endocrine disturbances accompanied by inflammatory changes. For instance, a number of studies have supported the association between low BMD and nonalcoholic fatty liver disease (NAFLD), which comprises a spectrum of disorders also involving liver inflammation. However, conflicting evidence regarding this association has been obtained thus far. ${ }^{8}$

This review will highlight the importance and newest developments on the relationship between NAFLD and osteoporosis.

\section{NAFLD}

The incidence of NAFLD in the community is rising, in parallel with the epidemics of type 2 diabetes and obesity as well as metabolic syndrome. ${ }^{9}$ A microscopic picture of NAFLD is characterized by significant lipid deposition in the hepatocytes of the liver parenchyma similar to changes usually seen in alcohol-induced liver injury. However, it must be pointed out that NAFLD may be considered only in those who do not have a history of alcohol abuse, use of steatogenic medication, or hereditary disorders, eg, Wilson's disease. NAFLD encompasses a broad spectrum of histological impairments, ranging from simple macrovesicular steatosis to steatohepatitis, advanced fibrosis, and cirrhosis. ${ }^{9,10}$ Therefore, it is further divided into NAFLD and nonalcoholic steatohepatitis (NASH). NAFLD is defined as the presence of hepatic steatosis with the absence of hepatocellular injury (ballooning of the hepatocytes). NASH is defined as the presence of hepatic steatosis and inflammation with hepatocyte injury (ballooning) with or without fibrosis. Patients diagnosed with NAFLD progressing to cirrhosis are being increasingly recognized as a major cause of liver-related morbidity and mortality. NAFLD is listed among the top three causes of liver transplantation due to its potential to progress to liver failure. The usual method to diagnose NAFLD has been ultrasonography, sometimes coupled with elevated liver enzymes; however, none of the imaging methods, such as computerized tomography and magnetic resonance, reliably assess steatohepatitis and fibrosis in NAFLD. Moreover, noninvasive biomarkers for identifying steatohepatitis in patients with NAFLD are also lacking to date. ${ }^{9-11}$

Although a vast number of intra- and extrahepatic factors influencing the development of fatty liver have been described, the pathogenesis of NAFLD has not been fully elucidated. ${ }^{9}$ However, among the factors that may lead to the development of NAFLD, complex interactions among genome, selected environmental factors, as well as the gut microbiome are considered to be the most important. It is also known that sterile cell death, as an outcome of lipotoxicity in hepatic cells, in parallel with altered gut-liver axis function encompassing translocation of bacterial products into portal circulation as a result of the so-called "leaky gut" may trigger the inflammation cascade that in consequence may lead to the development of NASH. It was demonstrated in both animals and humans that gut permeability in NAFLD is impaired and that the presence of NASH is strongly associated with altered gut permeability, which leads to the conclusion that liver injury and inflammation may be dependent on the degree of interruption of gut barrier integrity. ${ }^{12,13}$ Also of importance here, several studies have shown that major tight junction proteins are downregulated in patients with NAFLD and obesity by itself is associated with increased intestinal permeability. ${ }^{13}$ 
In recent epidemiological studies, NAFLD was shown to be connected with diseases that are usually not dependent on obesity, such as sarcopenia and osteoporosis. ${ }^{10}$ It is noteworthy that these diseases are significantly linked to aging as well as to NAFLD, which also tends to increase with age in men and women, independent of menopausal status. However, consequences of obesity, such as NAFLD, may also affect bone mineral acquisition in pediatric patients, which raises the hypothesis of other potential factors linking liver and bone tissue other than those which are commonly considered as age dependent. ${ }^{11,14,15}$

Most of the studies describing mutual relationships between NAFLD and bone mass are from Asia. In Chinese populations, a negative association was found between NAFLD and BMD in both postmenopausal women and men in several examined sites of the skeleton. Nevertheless, in patients with NAFLD, only femoral neck BMD was significantly lower when compared with healthy controls. ${ }^{16,17}$ Similar results were also found in a population of Korean men, and surprisingly, a positive association between lumbar spine BMD and NAFLD in women was found. ${ }^{18,43}$ Although the abovementioned studies are of value, it is not clear whether the results can be extrapolated to other populations because there are some studies showing contrary results such as the study conducted in India where a positive association of NAFLD with BMD was found. ${ }^{19}$ The reason for this discrepancy may be that neither BMI nor body fat content was considered in the comparison of BMD between groups. ${ }^{10,19}$ Pediatric studies, however, show a negative correlation between fatty liver and bone mass clearly even after adjustment for body fat mass or high-sensitivity $\mathrm{C}$-reactive protein levels. It must be pointed out that NAFLD in children was MRI and/or biopsy-proven. ${ }^{20,21}$

Among the main pathophysiological factors linking NAFLD with decreased bone mass are vitamin D deficiency, growth hormone/insulin-like growth factor 1 (GH/IGF-1) axis disturbances, as well as chronic inflammatory process.

\section{What are the links between NAFLD and decreased bone mass? Vitamin D insufficiency}

The role of vitamin D in bone homeostasis is crucial and well described. Although its insufficiency causes a decrease in luminal calcium absorption which, due to secondary hyperparathyroidism, leads to increased bone turnover followed by a decrease in bone mass, data regarding the role of vitamin D supplementation in prevention of osteoporotic fractures are inconsistent. ${ }^{22}$ In several studies, vitamin D was ineffective in the prevention of fractures; however, in other studies, it was shown that combined supplementation of vitamin $\mathrm{D}$ and calcium in elderly patients with vitamin $\mathrm{D}$ deficiency and secondary hyperparathyroidism prevented osteoporotic fractures. ${ }^{23,24}$ Low serum levels of vitamin D usually were linked with low BMD and an increased risk of fractures. ${ }^{25}$ Similarly, in a number of studies, serum levels of 25-hydroxyvitamin D were lower in subjects with NAFLD or NASH when compared with healthy controls; therefore, its insufficiency has been claimed to play a role in the pathogenesis of NAFLD. ${ }^{26-28}$ The aforementioned influence of vitamin D status on NAFLD notwithstanding the effectiveness of vitamin D supplementation in prevention and/or treatment of NAFLD in humans has not been demonstrated yet. ${ }^{29}$ Similarly, in both human and animal studies, evidence for the causal role of vitamin $\mathrm{D}$ in the biogenesis of NAFLD is elusive; however, at least one animal model experiment revealed that artificial sunlight may be a good complementary therapy for NASH because of its positive effect on serum levels of both lipid transfer/metabolic proteins and vitamin D3. ${ }^{30}$ Moreover, other animal studies showed that vitamin $\mathrm{D}$ inhibits liver fibrosis by blocking the activation of hepatic stellate cell, thus lowering collagen deposition and extracellular matrix remodeling. ${ }^{31}$ Very interesting data came also from China, where Su et al showed that a high-fat diet is necessary but insufficient to induce robust hepatic steatosis in mice models, and an additional vitamin D deficiency as a second factor is needed. ${ }^{32}$ Also of importance is the finding that dietary vitamin D supplementation in NAFLD-like animal models was shown to decrease the progress of hepatic steatosis as well as the progress of other body indices of metabolic syndromes. ${ }^{32}$ This finding was also confirmed in other studies, which revealed that lifelong administration of high doses of vitamin D prevented diabetes in NOD mice. ${ }^{33}$

\section{GH/IGF-I axis}

Among other abnormalities, GH deficiency in adults is characterized by the prevalence of NAFLD or NASH. ${ }^{34}$ Moreover, it was shown that substitution with GH can, at least in part, reverse these conditions. ${ }^{34}$

Visceral fat, which is crucial in the development of NAFLD, can be significantly reduced by external GH. Moreover, GH directly decreases lipogenesis in hepatocytes. Both GH and IGF-1 are of great importance in hepatic physiology, mainly due to the finding that IGF-1 is the main mediator of GH activity in subjects with NAFLD. ${ }^{34}$ However, in hepatic cells, the actions of GH and IGF-1 are contrary because IGF-1 stimulates glucose uptake favoring insulin signaling, while 
$\mathrm{GH}$ induces lipolysis determining insulin resistance mediated by elevated free fatty acid levels. ${ }^{35}$ Studies performed in patients with NAFLD showed lower levels of IGF-1 compared with healthy controls, and also, a negative association of IGF-1 levels with the histological severity of NAFLD was seen. ${ }^{36}$ It was also confirmed by other authors who found a negative correlation between serum IGF-1 levels and liver hyperechogenicity. ${ }^{37}$

The role of IGF-1 in hepatocytes seems to be multidirectional because it takes part not only in lipid regulation but also acts as a factor that is capable of downregulating liver fibrosis. It was shown that IGF-1 induces cellular senescence and inactivates hepatic stellate cells. In animal models, treatment with external IGF-1 was shown to lower the progression of both NASH and cirrhosis. This could be of great importance when considering its future clinical application in humans because histology in NASH, apart from steatosis and inflammation, also encompasses focal necrosis and fibrosis. ${ }^{38}$ What is of importance here is that low levels of IGF-1 coincide with both NAFLD and NASH as well as with osteoporosis, which also presents a clinical basis for considering IGF-1 as a potential anabolic agent for the treatment of osteoporosis.

The overall pleiotropic activity of IGF-1 is characterized by its involvement in bone tissue growth maturation as well as in skeleton rebuilding. No matter for what reason a decline in somatotropic axis activity appears, it is usually followed by disturbances in bone metabolism leading to the development of osteoporosis. ${ }^{39,40}$ Several studies have shown that low levels of IGF-1 are associated with a greater risk of vertebrae and hip fractures because bone tissue is the second richest source of IGF-1, and IGF-1 directly promotes osteoblast differentiation and growth. ${ }^{41-44}$ It was shown in elderly women that recombinant human IGF-1 may directly increase osteoblastic function with only a minimal increase in bone resorption. ${ }^{45}$ Moreover, IGF-1 was also considered to be useful as an early marker for the risk of low bone mass and osteoporosis in premenopausal and postmenopausal women indicated by measuring serum levels of IGF-1 in women around 40 years old with BMD below 1.5 SD measured by DEXA. ${ }^{46}$ Last but not the least, GH itself was also shown to be capable of directly inhibiting osteoclast formation in bone marrow cultures in vitro. ${ }^{47,48}$ In human studies, recombinant human GH accelerated the rate of bone turnover improving bone metabolism in postmenopausal women. ${ }^{47}$

In summary, although recent data clearly indicate the crucial role of $\mathrm{GH} / \mathrm{IGF}-1$ axis signaling in the pathogenesis of both NAFLD and osteoporosis, direct cross-talk linking among hepatocytes and bone cells has not been described to date. Most clinical studies involving both adults and children have clearly shown that NAFLD is connected with more common occurrences of decreased BMD in comparison with age-, sex-, and BMI-matched healthy controls, but there are also studies showing the opposite results. ${ }^{14,49}$ However, the systematically increasing clinical and experimental evidence leads to the conclusion that NAFLD affects the pathogenesis of decreased BMD, possibly through the systemic release of multiple proinflammatory, procoagulant, and profibrogenic mediators. Last but not the least, NAFLD directly influences whole-body and hepatic insulin resistance. ${ }^{14}$

\section{Circulating molecules and chronic inflammatory process}

Apart from the abovementioned abnormalities, another feature linking NAFLD with osteoporosis seems to be the chronic inflammatory process. Although pathogenic factors and triggering factors may be different, most of the systemic inflammation pathways and mediators involved are common for both NASH and inflammatory osteoporosis. Among the number of immunocompetent cells, hepatic stellate cells as well as dendritic cells have the capability of generating proinflammatory signals that may lead to premature apoptosis, resulting in hepatocyte death and the development of NASH and cirrhosis. Cellular lipid overload resulting in cell lipotoxicity is one of the best recognized triggering factors for inflammation cascade process in hepatocytes. Moreover, the so-called "sterile" inflammation causes local injury, which activates fibroblasts that may lead to liver fibrosis. ${ }^{50}$ Among many of the molecules involved, tumor necrosis factor-alpha (TNF- $\alpha$ ) is considered to be one of the key factors that plays a role in both mediating and inducing hepatocyte death as well as a classical inducer of inflammatory osteoporosis.

\section{TNF- $\alpha$}

Jarrar et al examined the role of adipokines in the pathogenesis of NAFLD and showed that TNF- $\alpha$ level increases significantly in obese controls, patients with simple steatosis, and patients with NASH. ${ }^{51}$ This was also visible in other clinical studies, showing that serum levels of TNF- $\alpha$ in NAFLD patients are significantly increased. ${ }^{52}$ Moreover, in blood cells collected from obese patients with NAFLD, an increased production of TNF- $\alpha$ and interleukin (IL)-1 was shown. ${ }^{53}$ The role of TNF- $\alpha$ was also demonstrated in a clinical trial with pentoxifylline (a TNF- $\alpha$ inhibitor) that was performed in patients with $\mathrm{NASH}$, where a significant reduction of serum TNF- $\alpha$ followed by normalization of 
liver function was archived after 6 months of treatment. ${ }^{54}$ It is believed that the role of TNF- $\alpha$ in NAFLD pathogenesis is manifold; however, increasing SREBP-1c (sterol regulatory element binding protein-1c) dependent on intrahepatic fat deposition and attracting inflammatory leukocytes to the liver are considered to be most important. ${ }^{55} \mathrm{TNF}-\alpha$ also plays a crucial role in bone homeostasis by influencing complex signaling pathways leading to gene transcription of several regulators of bone homeostasis.

In the current literature, TNF- $\alpha$ is generally described as a factor inducing osteoclastogenesis in parallel with inhibition of osteoblastogenesis. However, there are also data showing that it may exert positive effects on osteoblast differentiation. ${ }^{56,57}$ This paradox is visible when analyzing the pathogenesis of osteoporotic changes in rheumatoid arthritis (RA) and ankylosing spondylitis (AS). Because in both conditions, serum TNF- $\alpha$ levels may be relatively high; in RA, inhibition of selected genes favors bone resorption, while in AS, apart from predominant bone resorption, characteristic ectopic bone formation is also visible. ${ }^{57}$ This indicates that TNF- $\alpha$ acts in multiple ways depending on the molecular pathway followed. Under inflammatory conditions, activated $\mathrm{T}$ cells and B cells secrete other pro-osteoclastogenic molecules apart from TNF- $\alpha$ like the receptor activator of nuclear factor- $\kappa \mathrm{B}(\mathrm{NF}-\kappa \mathrm{B})$ ligand (RANKL) and IL-17A, which leads to the induction of bone loss. Similarly, in postmenopausal osteoporosis and hyperparathyroidism, accelerated bone turnover is connected with increased secretion of TNF- $\alpha$ in parallel with IL-17A and RANKL by activated T cells. ${ }^{58}$ All the abovementioned processes indicate that inflammation links NAFLD with osteoporosis and emphasizes the role of TNF- $\alpha$ as well as other proinflammatory molecules like IL-6 and macrophage colony-stimulating factor. However, there are also some other independent potential cofounders that should be mentioned.

\section{OPG}

As it was mentioned in the first section of this review, a completely new understanding of both differentiation and activation of osteoclasts appeared after the discovery of the RANK/RANKL/osteoprotegerin (OPG) pathway. The association of OPG and fragility fractures still remains controversial because data from clinical studies are inconsistent, eg, in some studies a positive correlation of OPG and BMD in both women and men was noticeable, whereas other studies showed the opposite result. ${ }^{59-61}$ However, the leading role of OPG in maintaining bone homeostasis remains unquestionable. Thus, the profound elucidation of OPG biology provided an opportunity for its application in the treatment of bone metabolic disturbances, especially those with excessive bone resorption. ${ }^{56}$ What is of importance here is that the biological activity of OPG is not limited solely to bone tissue but is also expressed and acts in other cells and tissue types, eg, T lymphocytes and large arteries where OPG may play an important role in both development and function of these cells and tissues. ${ }^{62}$ Regarding metabolic syndromes, an attempt at elucidation of the possible function of OPG in the cross talk between NAFLD and bone tissue was made by Yilmaz et al. They have shown that in patients with NASH and borderline NASH, serum OPG concentrations are reduced in patients with more severe forms of NAFLD and may serve as a noninvasive biomarker to identify patients with NASH. ${ }^{63}$ This was further confirmed by Chinese researchers, who found that serum OPG level was significantly lower in NASH patients than in non-NASH patients and that this was highly correlated with hepatocyte fatty degeneration, ballooning degeneration, intralobular inflammation, portal inflammation, as well as with fibrosis degree. ${ }^{64,88}$ Similar results were also found in patients with type 2 diabetes, where Niu et al have shown that plasma OPG levels are negatively associated with NAFLD. ${ }^{65}$

\section{Fetuin-A}

The plasma protein $\alpha 2$-Heremans-Schmid glycoprotein/ fetuin-A is a $66 \mathrm{kDa}$ glycoprotein that together with IGFs, insulin-like growth factor-binding proteins (IGFBPs), selenoprotein $\mathrm{P}$, sex hormone-binding globulin, and fibroblast growth factor-21 (FGF-21) is a member of a family of proteins called hepatokines exclusively secreted by the liver into circulation. These molecules have multiple biological functions among which the most important seems to be modulating insulin sensitivity, which makes them able to serve as biomarkers of insulin resistance and related metabolic disorders, such as NAFLD. ${ }^{66}$ The fetuin-A gene is located on chromosomal $3 \mathrm{q} 27$, whose expression is found to be associated with metabolic syndrome and type 2 diabetes, which is confirmed by the fact that fetuin-A positively correlates with metabolic syndrome and insulin resistance. ${ }^{67,68}$ A Chinese study by Cui et al showed that the serum levels of fetuin-A were lower in NAFLD patients than those in controls, while fetuin-A levels increased with the severity of NAFLD. They also indicated fetuin A as a potential biomarker in the development of NAFLD. ${ }^{69} \mathrm{~A}$ similar study confirmed that circulating fetuin-A level is elevated in individuals with hepatic fat accumulation and NAFLD $;{ }^{70}$ however, there is also at least one other study that gave 
opposite results. ${ }^{71}$ There is also increasing evidence showing that fetuin-A is elevated in bone tissue and acts as a mediator of formation of stable colloidal mineral-protein complexes called calciprotein particles, which is of importance in the stabilization and clearance of amorphous mineralization. Moreover, apart from being an important inhibitor of ectopic calcification, it has been described to influence the production of mediators of inflammation. Furthermore, a regulatory role in bone metabolism has also been described in the case of fetuin-A deficiency (Ahsg(-/-)), where impaired growth of long bones and premature growth plate closure were shown. Fetuin-A exhibits its action as a carrier for lipids, which may influence calcification, inflammation, and apoptosis. ${ }^{72}$ Because some authors showed that in older women, serum fetuin-A is dependent on both bone mass and bone resorption markers and may be significantly decreased after cessation of menses, it was postulated that it can serve as a marker of bone mineralization. ${ }^{73,74}$ Thus, considering all the abovementioned features regarding the role of fetuin-A in both NAFLD and bone metabolism, one might conclude that lower serum levels of fetuin-A in NAFLD patients may be an important factor leading to a decrease of bone mass. However, the clinical significance of this hypothesis has not been fully elucidated up to date.

\section{Osteopontin (OPN)}

OPN, also defined as secreted phosphoprotein-1 (SPP1), sialoprotein-1, early $\mathrm{T}$ lymphocyte activation 1 (Eta-1), belongs to the small integrin-binding ligand N-linked glycoprotein (SIBLING) family. Physiologically, it is thought to regulate biomineralization in bone tissue and to reduce growth and aggregation of calcium crystals in epithelial tissues; however, in the past 10 years, OPN has been shown to be an important factor in various processes, such as, inflammatory and immune response, malignancy, type 2 diabetes, and angiogenesis. The highest abundance of OPN was detected in bone tissue comprising approximately $2 \%$ of the noncollagenous organic matrix. The biological role of this 300 -amino acid molecule is to mediate signal transduction by binding to the cell surface receptors integrin $\alpha v \beta 3$ and CD44. It is detectable in almost all body fluids, and in bone tissue it is secreted by both osteoblasts and osteoclasts. OPN in bone tissue stimulates migration and adhesion of osteoclasts to the bone matrix through the interaction of $\alpha v \beta 3$ and CD44 and increases CD44 expression on osteoclasts, which may lead to resorption because CD44 expression is obligatory for osteoclasts activity. ${ }^{75,76}$ The role of OPN in osteoporosis has been thoroughly studied in both human and animal models, and it has been shown that OPN-deficient mice are resistant to ovariectomy-induced osteoporosis. ${ }^{76}$ In postmenopausal women, elevated levels of OPN were shown to be an important risk factor for the development of osteoporosis. ${ }^{77}$

Because it became evident that osteoporosis and obesity are two related polygenic disorders, numerous studies have been conducted to clarify if a relationship between obesity and/or NAFLD and osteoporosis exists. ${ }^{78-80}$ Because high levels of OPN RNA expression in adipose tissue of obese insulin-resistant rats and humans were described, some authors have suggested that OPN may be the factor that directly links obesity-induced inflammation with metabolic disturbances. ${ }^{81-83}$ Indeed, it was shown that, within the liver, increased OPN expression is present not only in hepatocytes but also primarily in inflammatory cells. ${ }^{84}$ Moreover, animal model experiments demonstrated that in the liver of obese mice, OPN expression is significantly upregulated and that hepatic OPN levels correlate with liver triacylglycerol levels. ${ }^{80}$ Further studies performed in mice fed methionine- and choline-deficient diet also shed more light on the role of OPN in the development of NAFLD. ${ }^{85,86}$ Other studies showed that antibody-mediated OPN neutralization and OPN deficiency may exert a protective effect against HFD-induced hepatic macrophage infiltration and D-galactosamine-induced inflammatory liver injury. ${ }^{80,84}$ Taken together, the abovementioned experiments indicate that the OPN molecule links obesity-associated hepatic inflammation, NAFLD, and insulin resistance. However, on the contrary, it clearly demonstrated that OPN-deficient mice are resistant to ovariectomy-induced osteoporosis. ${ }^{85-87}$ Therefore, one could speculate that OPN could not only be a target for therapies against metabolic syndrome but also due to the fact that its overexpression can be present in NAFLD, it may be associated with less resistance to postmenopausal bone loss and osteoporosis.

\section{Osteocalcin (OC)}

Serum OC produced and released by osteoblasts in bone tissue is traditionally considered one of the markers of bone formation. Although most studies have focused on its role as a predictor of fractures, in recent years OC has been recognized as a molecule that may have the potential for regulating energy metabolism and glucose tolerance. For example, it was shown in OC-deficient mice with glucose intolerance and insulin resistance induced by a high-fat diet that external supplementation with recombinant OC improved metabolic derangement. ${ }^{88}$ The underlying mechanism has not been fully elucidated yet; however, Du et al suggested that therapeutic 
effects of OC in NAFLD may be intervened through activation of the nuclear factor like-2 (Nrf-2) pathway to alleviate oxidative stress and inhibition of the C-Jun N-terminal kinase (JNK) pathway in the liver. ${ }^{89}$ In addition, there are studies showing that $\mathrm{OC}$ improves glucose intolerance via production of adiponectin in adipose tissue and enhancement of insulin secretion. ${ }^{90}$ In humans, serum OC levels correlate with glucose intolerance, insulin resistance, and obesity, as well as with metabolic syndrome. ${ }^{91,92}$ It was demonstrated by Zhou et al that improvement of insulin resistance by OC is probably mediated by attenuation of endoplasmic reticulum stress via inhibition of the NF- $\mathrm{KB}$ pathway. ${ }^{93}$ However, it must be pointed out that although there are many animal data about the role of OC on glucose metabolism, the results of human clinical data are inconsistent, and the role of $\mathrm{OC}$ in human remains controversial.

Yilmaz et al found that patients with biopsy-proven NAFLD have significant reductions in serum OC concentrations (vs matched controls), which were weak, but significantly associated with the extent of hepatocyte ballooning, independent of insulin resistance and metabolic syndrome. ${ }^{94}$ This was also elaborated in pediatric patients, where Amin et al demonstrated that OC plays an important role in glucose and lipid metabolism for protection against NAFLD occurrence and progression. Moreover, it could be a useful marker for progression of NAFLD in children with obesity. ${ }^{95}$ Most epidemiological studies suggest that a lower OC level is a risk factor for NAFLD. ${ }^{96,97}$ Because it is commonly accepted that reduced OC levels are associated with increased bone loss, it is reasonable to assume that in those with NAFLD, lower OC concentrations, among others, could also be one of the factors that links hepatic steatosis and reduced bone mass. ${ }^{56}$

\section{Leptin and adiponectin}

Other mediators of specific cross talk between the liver, adipose tissue, and bone, apart from TNF- $\alpha$, are leptin and adiponectin. Both molecules are considered to be involved in neuroendocrine control of energy balance and are primarily expressed by adipose tissue, and their role in the pathogenesis of NAFLD is well established. For example, several studies have shown that animals deprived of leptin expression (ob/ob mice) are insulin resistant, obese, and develop NAFLD, where alterations are reversed by leptin administration. ${ }^{98,99}$ Similarly, it was shown in animal models of NASH that external adiponectin administration may, through insulin-sensitizing, antilipogenic, anti-inflammatory, and anti-fibrogenic modes of action, significantly improve metabolic profile and liver histology. ${ }^{100}$ In terms of other known adipokines, eg, visfatin, chemerin, retinol-binding protein 4, resistin, and irisin, available data on their role in NAFLD development are ambiguous. ${ }^{98}$ Adiponectin deficiency is associated with a proinflammatory condition, as it is observed in obesity and other metabolic disorders. Increased leptin levels, however, act as a proinflammatory stimulus. The adiponectin/leptin ratio is therefore considered as a better inflammatory biomarker of inflammation in metabolic syndrome patients than these adipokines analyzed in isolation. ${ }^{101}$ In obese humans, hyperleptinemia is associated with a reduction of bone formation biomarkers, especially OC. ${ }^{102}$ On the contrary, adiponectin seems to improve bone formation, ${ }^{103}$ promoting the proliferation, differentiation, and mineralization of osteoblastic cells. ${ }^{104}$

Leptin may influence the regulation of bone metabolism directly by increasing central sympathetic activity by binding to its receptors on both the hypothalamic ventromedial (VMH) nucleus and the serotoninergic brainstem neurons, which in turn project to VMH neurons. ${ }^{105}$ Sympatric fibers then transmit signals to osteoblasts through the beta-2adrenergic receptor, which inhibits osteoblast differentiation and the generation of OC. ${ }^{106,107}$ It was also shown that leptin may directly stimulate osteoblast differentiation and bone mineralization via its receptors present on osteoblasts. ${ }^{108}$ Moreover, leptine's mode of action also includes stimulation of hepatic IGF-binding protein 2 (IGFBP-2) secretion which, apart from improving the insulin sensitivity, enhances osteoblast differentiation. ${ }^{107,109}$

In several studies, adiponectin was shown to stimulate bone formation and remodeling as well as inhibit bone resorption, which suggests that it may be considered as a negative regulator of bone mass. Other studies, however, demonstrate that adiponectin stimulates the differentiation and mineralization of osteoblasts and enhances RANKL secretion as well as the expression of $\mathrm{OC}$, which acts as a hormone-regulating glucose metabolism and fat mass. ${ }^{103,110-112}$ In osteoclasts, adiponectin inhibits RANKL-mediated osteoclastogenesis through interaction with its adaptor molecule APPL1 (adaptor protein containing pleckstrin homology domain, phosphotyrosine domain, and leucine zipper motif). ${ }^{113}$ The results from human studies are not so consistent. ${ }^{114-116}$ For instance, in a cohort of 365 older adults, high levels of adiponectin and adiponectin to leptin ratios were both associated with lower BMD at the lumbar spine and femoral neck, ${ }^{115}$ but other studies do not indicate such associations. ${ }^{116}$ But it must be pointed out that the observed discrepancy in the influence of adiponectin on osteoblasts and osteoclasts and consequently on bone remodeling may be the result of the 
complex interrelationship between the endocrine system (including leptin) and the fat metabolism.

\section{Insulin resistance and physical activity}

The role of insulin resistance in the pathogenesis of hepatic steatosis is well described, and recent in vitro and in vivo studies indicate that insulin may also play an important role in the homeostasis of bone tissue. Insulin impacts the bone microarchitecture and mechanical properties by increasing collagen synthesis and also by promoting osteoblast proliferation and differentiation. ${ }^{117}$ Traditionally, it was believed that obesity has a protective effect against bone loss; however, some uncertainty appeared because some studies have demonstrated that the relationship between metabolic syndrome and bone strength or BMD is negative in models adjusted for BMI. ${ }^{118}$ Animal model studies demonstrated that insulin signaling in bone tissue is mediated by osteoblasts and that, apart from muscle and liver, indicate that the skeleton also is a site of insulin resistance. Moreover, OC seems to be a key signaling molecule because it was shown that bone OC may influence whole-body insulin sensitivity. ${ }^{119,120}$ In addition, there are studies clearly indicating that BMD and the presence of insulin resistance are negatively correlated. ${ }^{121}$

Physical activity is currently considered to be essential in the prevention of both osteoporosis and NAFLD. Various training studies have shown that exercise may reduce the risk of fragility fractures in postmenopausal women. ${ }^{122}$ Similarly, physical activity reduces the levels of visceral adiposity in the absence of weight loss, which might be a key mechanism in protection from NAFLD. ${ }^{123}$ Although the mechanisms remain poorly understood, it is reasonable to conclude that low levels of physical activity lead to a decrease in BMD, the development of insulin resistance, and as a consequence to the presence of NAFLD. The exact mechanism of exerciseinduced liver fat reduction is unknown, but it was found that irisin may be involved because plasma irisin negatively correlates with intrahepatic triglyceride content, which was demonstrated in Chinese patients with NAFLD. ${ }^{124}$ The name irisin derives from the Greek Goddess Iris (messenger of the gods), and it is a newly described member of the myokine family, whose levels seem to increase during physical exercise leading to heat expenditure and a possible protective effect on metabolic disorders, including NAFLD. It was found that irisin may act as endocrine factor influencing the activity of both osteoblasts and adipocytes. Moreover, it is also capable of regulating the expression of osteogenic genes and inducing differentiation of osteoblasts. Although more research on the biological role of irisin is needed before firm conclusions can be drawn, it appears that there is a chance, at least because there are available data describing its multiple effects on obesity, insulin resistance, and bone tissue, that irisin might be the lacking element that links bone and liver metabolism. ${ }^{125,126}$

\section{Marrow adipose tissue (MAT)}

MAT is functionally distinct from both white and brown adipose but can contribute to systemic and skeletal metabolism. In young healthy individuals, MAT was not associated with any measure of metabolic risk, including waist-to-hip ratio, blood pressure, carotid intima-media thickness, insulin resistance, or lipid profile, ${ }^{127}$ suggesting that in young, healthy individuals MAT is metabolically distinct from visceral adipose tissue. However, such association was noticed in obese women as well as in patients with type 2 diabetes. ${ }^{128}$ Because noninvasive methods of measuring MAT (such as magnetic resonance spectroscopy) are available, it was shown in clinical studies of individuals with metabolic disease that MAT can be inversely associated with measures of BMD and bone integrity and therefore may be an important regulator of bone turnover. ${ }^{129,130}$ Although the underlying mechanisms are not fully elucidated yet, it is generally accepted that MAT arises in bone marrow from mesenchymal stem cells that are more closely related to osteoblasts than other cells of mesenchymal origin, eg, stromal cells or chondrocytes. Although some animal studies indicate that increased adipogenesis can be an inhibitor of bone marrow hematopoiesis, other studies revealed that interactions between marrow adipocytes and hematopoiesis are more complex. For example, in mice with very high marrow adiposity $(\mathrm{C} 3 \mathrm{H} / \mathrm{HeJ}$ mice), intact hematopoiesis and high cortical bone density were shown. ${ }^{130}$ On the contrary, in Ebf1 knockout mice with B-cell deficiency, high bone marrow fat content followed by depletion of the hematopoietic system was noticed. ${ }^{131}$ Among potential determinants of MAT, early B-cell factor 1 (Ebf1) and preadipocyte factor-1 (Pref-1) are currently the best recognized. Ebf1, originally described as a signaling molecule necessary for B-lymphocyte maturation, was also shown to influence the development of other cells, including both osteoblasts and adipocytes. ${ }^{132}$ Similarly, Pref-1, a member of the epidermal growth factor-like family of proteins, is a known regulator of adipocyte and osteoblast differentiation. ${ }^{133,134}$ In summary, although in the light of a number of animal experiments, MAT may be considered a central regulator of skeletal energy allocation between bone turnover and hematopoiesis; however, data from human studies on the function of marrow adipocytes are still sparse. 


\section{Klotho gene family and fibroblast growth factors}

As it was mentioned above, soluble and membrane-bound Klotho functions as a receptor for a hormone that regulates excretion of phosphate as well as kidney synthesis of active vitamin $\mathrm{D}$ and is encoded by $\alpha$-Klotho $(\mathrm{KL} \alpha)$, originally identified as an antiaging gene. Although various functional aspects of Klotho and FGF-23 have been investigated that lead to the identification of multiple novel endocrine axes that regulate various metabolic processes including bone metabolism, there are no data regarding the possible contribution of KL $\alpha$ to metabolic syndrome or NAFLD alone. ${ }^{135-137}$ Recently, a new form of the Klotho gene (and its protein products), $\beta$-Klotho (Klb) was discovered. As a coreceptor mediating FGF-21 and FGF-19 signaling, Klb, apart from glucose utilization regulation, thermogenesis as well as bile acid homeostasis, it may also play an important role in hepatic fat deposition. The Klb transmembrane protein is expressed in the liver, pancreas, and adipose tissue and together with FGF-21 is secreted from liver upon fasting and acts on adipose tissue to promote lipolysis in response. ${ }^{138,139}$ Further studies on the endocrine axes mediated by the Klotho family and FGF-19, FGF-21 and FGF-23 subfamily members are needed to elucidate molecular mechanisms by which the endocrine fibroblast growth factors regulate bile acid, energy, and glucose utilization as well as phosphate/ vitamin D metabolism.

\section{Aging}

Population aging is a challenge for clinicians because the number of elderly patients is growing with age-associated comorbidities, many of them very costly, as is the case of cardiovascular diseases, cancer, as well as osteoporosis, and metabolic syndrome. NAFLD is expected to play most important role among age-related liver diseases in the setting of the secular worldwide trend toward an increment of metabolic diseases, notably including those associated with reduced insulin sensitivity. Moreover, in the elderly, both NAFLD and osteoporosis carry more substantial burden of complications, eg, NASH, cirrhosis, hepatocellular carcinoma, and increased number of fragility fractures. ${ }^{140}$ Recent data clearly indicate that both advanced age and body fat mass are positively correlated with the risk of osteoporotic fracture. ${ }^{141,142}$ Possible mechanisms specifically linking alterations of bone metabolism and fractures with NAFLD in elderly are not clearly elucidated yet. However, both chronic inflammation and oxidative stress accompanying hepatocyte fat overload may also at the same time negatively influence bone metabolism. ${ }^{143,144}$ What is of importance here is that most previous studies on fracture risk have been performed in patients with chronic cholestatic liver diseases or liver transplant recipients. There are only three available cross-sectional studies which exclusively focused on the association between presence of NAFLD and osteoporotic fractures in elderly. Although all were performed in Asian populations, the results obtained were inconsistent. Li et al ${ }^{144}$ showed a positive correlation of NAFLD with fracture risk with the magnitude of the association similar to the previously observed relationships of osteoporotic fracture with other chronic liver diseases ${ }^{145-147}$ in men, but not in women. ${ }^{144}$ Surprisingly, in other studies, such associations were not detected in men, and the presence of metabolic syndrome was significantly associated with a recent history of osteoporotic fracture in both middle-aged and elderly women. ${ }^{148,149}$ Therefore, both population- and sex-specific associations of NAFLD with osteoporotic fracture need to be further elucidated.

\section{Conclusion}

Apart from the traditional risk factors of osteoporosis, such as old age, menopause, or decreased BMI, insulin resistance and metabolic syndrome are also currently listed among factors associated with decreased BMD. NAFLD is the most common chronic liver disease associated with insulin resistance and metabolic derangement, and recently its pathogenesis irrespective of age and gender resulting in increased bone loss in patients was intensively studied. In this review, various pathophysiological mechanisms and possible mediating molecules that may interplay between NAFLD and bone tissue have been described. Chronic inflammation, vitamin D3, GH, IGF-1, OPN, fetuin-A, irisin, OC, and OPG from osteoblasts have been proposed as mediators of mutual interactions among the skeleton, fatty tissue, and liver. Although there are still many issues that have not been elucidated up to date, growing evidence suggests that screening and surveillance of BMD in patients with NAFLD may be considered in future strategies and in guidelines for NAFLD management. Moreover, promising findings in some of the clinical studies on the efficacy of vitamin D in patients with NAFLD justify its supplementation during the course of disease.

\section{Disclosure}

The authors report no conflicts of interest in this work

\section{References}

1. Kanis JA. WHO Technical Report. Vol. 66. United Kingdom: University of Sheffield; 2007. 
2. Gullberg B, Johnell O, Kanis JA. World-wide projections for hip fracture. Osteoporos Int. 1997;7(5):407-413.

3. Yang Y, Wang B, Fei Q, et al. Validation of an osteoporosis selfassessment tool to identify primary osteoporosis and new osteoporotic vertebral fractures in postmenopausal Chinese women in Beijing. BMC Musculoskelet Disord. 2013;14(1):271-274.

4. Katz S, Weinerman S. Osteporosis and gastrointestinal disease. Gastroenterol Hepatol (NY). 2010;6:506-517.

5. Katz S. Osteoporosis in patients with inflammatory bowel disease: risk factors, prevention, and treatment. Rev Gastroenterol Disord. 2006; 6:63-71.

6. Larsen S, Bendtzen K, Nielsen OH. Extraintestinal manifestations of inflammatory bowel disease: Epidemiology, diagnosis, and management. Ann Med. 2010;42(2):97-114.

7. Levine JS, Burakoff R. Extraintestinal manifestations of inflammatory bowel disease. Gastroenterol Hepatol (NY). 2011;7:235-241.

8. Upala S, Jaruvongvanich V, Wijarnpreecha K, Sanguankeo A. Nonalcoholic fatty liver disease and osteoporosis: a systematic review and meta-analysis. J Bone Miner Metab. 2017;35(6):685-693.

9. Sass DA, Chang P, Chopra KB. Nonalcoholic fatty liver disease: a clinical review. Dig Dis Sci. 2005;50:171-180.

10. Poggiogalle E, Donini LM, Lenzi A, Chiesa C, Pacifico L. Nonalcoholic fatty liver disease connections with fat-free tissues: A focus on bone and skeletal muscle. World J Gastroenterol. 2017;23(10): $1747-1757$.

11. Chalasani N, Younossi Z, Lavine JE, et al. The diagnosis and management of non-alcoholic fatty liver disease: practice Guideline by the American Association for the Study of Liver Diseases, American College of Gastroenterology and the American Gastroenterological Association. Am J Gastroenterol. 2012;107(6):811-826.

12. Kirpich IA, Marsano LS, Mcclain CJ. Gut-liver axis, nutrition, and non-alcoholic fatty liver disease. Clin Biochem. 2015;48(13-14): 923-930.

13. Teixeira TFS, Collado MC, Ferreira CLLF, Bressan J, Peluzio Mdo C. Potential mechanisms for the emerging link between obesity and increased intestinal permeability. Nutr Res. 2012;32(9):637-647.

14. Targher G, Lonardo A, Rossini M. Nonalcoholic fatty liver disease and decreased bone mineral density: is there a link? $J$ Endocrinol Invest. 2015;38(8):817-825.

15. Bertolotti M, Lonardo A, Mussi C. Nonalcoholic fatty liver disease and aging: Epidemiology to management. World J Gastroenterol. 2014; 20(39):14185-14204.

16. Xia M-F, Lin H-D, Yan H-M, et al. The association of liver fat content and serum alanine aminotransferase with bone mineral density in middle-aged and elderly Chinese men and postmenopausal women. J Transl Med. 2016;14(1):11.

17. Cui R, Sheng H, Rui X-F, et al. Low bone mineral density in Chinese adults with nonalcoholic fatty liver disease. Int J Endocrinol. 2013; 2013(1):396545-396546.

18. Lee SH, Yun JM, Kim SH, et al. Association between bone mineral density and nonalcoholic fatty liver disease in Korean adults. J Endocrinol Invest. 2016;39(11):1329-1336.

19. Bhatt SP, Nigam P, Misra A, Guleria R, Qadar Pasha MA. Independent associations of low 25 hydroxy vitamin $\mathrm{D}$ and high parathyroid hormonal levels with nonalcoholic fatty liver disease in Asian Indians residing in north India. Atherosclerosis. 2013;230(1):157-163.

20. Pacifico L, Bezzi M, Lombardo CV. Adipokines and C-reactive protein in relation to bone mineralization in pediatric nonalcoholic fatty liver disease. World J Gastroenterol. 2013;19(25):4007-4014.

21. Pardee PE, Dunn W, Schwimmer JB. Non-alcoholic fatty liver disease is associated with low bone mineral density in obese children. Aliment Pharmacol Ther. 2012;35(2):248-254.

22. Reid IR, Bolland MJ, Grey A. Effects of vitamin D supplements on bone mineral density: a systematic review and meta-analysis. Lancet. 2014;383(9912):146-155.

23. Francis RM. Calcium, vitamin D and involutional osteoporosis. Curr Opin Clin Nutr Metab Care. 2006;9:13-17.
24. Lips P, Bouillon R, van Schoor NM, et al. REVIEW ARTICLE: Reducing fracture risk with calcium and vitamin D. Clin Endocrinol. 2010; 73(3):277-285.

25. Lips P. Vitamin D deficiency and secondary hyperparathyroidism in the elderly: consequences for bone loss and fractures and therapeutic implications. Endocr Rev. 2001;22(4):477-501.

26. Wang X, Li W, Zhang Y, Yang Y, Qin G. Association between vitamin D and non-alcoholic fatty liver disease/non-alcoholic steatohepatitis: results from a meta-analysis. Int J Clin Exp Med. 2015;8:17221-17234.

27. Eliades M, Spyrou E, Agrawal N, et al. Meta-analysis: vitamin D and non-alcoholic fatty liver disease. Aliment Pharmacol Ther. 2013;38(3): 246-254.

28. Kwok RM, Torres DM, Harrison SA. Vitamin D and nonalcoholic fatty liver disease (NAFLD): Is it more than just an association? Hepatology. 2013;58(3):1166-1174.

29. Barchetta I, del Ben M, Angelico F, et al. No effects of oral vitamin D supplementation on non-alcoholic fatty liver disease in patients with type 2 diabetes: a randomized, double-blind, placebo-controlled trial. BMC Med. 2016;14(1):92.

30. Nakano T, Cheng Y-F, Lai C-Y, et al. Impact of artificial sunlight therapy on the progress of non-alcoholic fatty liver disease in rats. J Hepatol. 2011;55(2):415-425.

31. Abramovitch S, Sharvit E, Weisman Y, et al. Vitamin D inhibits development of liver fibrosis in an animal model but cannot ameliorate established cirrhosis. Am J Physiol Gastrointest Liver Physiol. 2015;308(2):G112-G120.

32. Su D, Nie Y, Zhu A, et al. Vitamin D signaling through induction of Paneth cell defensins maintains gut microbiota and improves metabolic disorders and hepatic steatosis in animal models. Front Physiol. 2016;15:498.

33. Takiishi T, Ding L, Baeke F, et al. Dietary supplementation with high doses of regular vitamin $\mathrm{D}_{3}$ safely reduces diabetes incidence in NOD mice when given early and long term. Diabetes. 2014;63(6): 2026-2036.

34. Takayashi Y. The role of growth hormone and insulin-like growth factor-I in the liver. Int J Mol Sci. 2017;18:1441.

35. Aguirre GA, de Ita JR, de La Garza RG, Castilla-Cortazar I. Insulinlike growth factor-1 deficiency and metabolic syndrome. J Transl Med. 2016;14(1):3.

36. Sumida Y, Yonei Y, Tanaka S, et al. Lower levels of insulin-like growth factor-1 standard deviation score are associated with histological severity of non-alcoholic fatty liver disease. Hepatol Res. 2015;45(7):771-781.

37. Völzke H, Nauck M, Rettig R, et al. Association between hepatic steatosis and serum IGF1 and IGFBP-3 levels in a population-based sample. Eur J Endocrinol. 2009;161(5):705-713.

38. Takahashi Y. Essential roles of growth hormone $(\mathrm{GH})$ and insulin-like growth factor-I (IGF-I) in the liver. Endocr J. 2012;59(11):955-962.

39. Perrini S, Laviola L, Carreira MC, Cignarelli A, Natalicchio A, Giorgino F. The GH/IGF1 axis and signaling pathways in the muscle and bone: mechanisms underlying age-related skeletal muscle wasting and osteoporosis. J Endocrinol. 2010;205(3):201-210.

40. Bikle DD, Tahimic C, Chang W, Wang Y, Philippou A, Barton ER. Role of IGF-I signaling in muscle bone interactions. Bone. 2015;80:79-88.

41. Sugimoto T, Nishiyama K, Kuribayashi F, Chihara K. Serum levels of IGF-I, IGFBP-2, and IGFBP-3 in osteoporotic patients with and without spine fractures. J Bone Miner Res. 1997;12:1272-1279.

42. Bauer DC, Rosen C, Cauley J, Cummings SR. Low serum IGF-I but not IGFBP-3 predicts hip and spine fracture: the study of osteoporotic fracture. J Bone Miner Res. 1998;23:S561.

43. Donahue LR, Rosen CJ. IGFs and bone. The osteoporosis connection revisited. Proc Soc Exp Biol Med. 1998;219:1-7.

44. Okman-Kilic T, Sagiroglu C. Anabolic Agents as New Treatment Strategy in Osteoporosis, Topics in Osteoporosis; 2013. Available from: http://www.intechopen.com/embed/topics-in-osteoporosis/ anabolic-agents-as-new-treatment-strategy-in-osteoporosis. Accessed September 7, 2018. 
45. Ghiron L, Thompson J, Halloway L, Butterfield GE, Hoffman A, Marcus R. Effects of rhGH and IGF-I on bone turnover in elderly women. $J$ Bone Miner Res. 1995;10:1844-1852.

46. Liu J-Min, Zhao H-Yan, Ning G, et al. IGF-1 as an early marker for low bone mass or osteoporosis in premenopausal and postmenopausal women. J Bone Miner Metab. 2008;26(2):159-164.

47. Ohlsson C, Bengtsson BA, Isaksson OGP, Andreassen TT, Slootweg M. Growth hormone and bone. Endocr Rev. 1998;19(1):55-79.

48. Ransjö M, Lerner U, Ohlsson C. Growth hormone inhibits formation of osteoclast like cells in mouse bone marrow cultures. J Bone Miner Res. 1996;11:T394.

49. Kaya M, Iş̧1k D, Beştaş R, et al. Increased bone mineral density in patients with nonalcoholic steatohepatitis. World J Hepatol. 2013;5(11) $627-634$

50. Seki E, Schwabe RF. Hepatic inflammation and fibrosis: Functional links and key pathways. Hepatology. 2015;61(3):1066-1079.

51. Jarrar MH, Baranova A, Collantes R, et al. Adipokines and cytokines in non-alcoholic fatty liver disease. Aliment Pharmacol Ther. 2008; 27(5):412-421.

52. Chu CJ, Rh L, Wang SS, et al. Risk factors associated with non-alcoholic fatty liver disease in Chinese patients and the role of tumor necrosis factor-alpha. Hepatogastroenterology. 2007;54:2099-2102.

53. Poniachik J, Csendes A, Díaz JC, et al. Increased production of IL- $1 \alpha$ and TNF- $\alpha$ in lipopolysaccharide-stimulated blood from obese patients with non-alcoholic fatty liver disease. Cytokine. 2006;33(5): 252-257.

54. Satapathy SK, Garg S, Chauhan R, et al. Beneficial effects of tumor necrosis factor-alpha inhibition by pentoxifylline on clinical, biochemi$\mathrm{cal}$, and metabolic parameters of patients with nonalcoholic steatohepatitis. Am J Gastroenterol. 2004;99(10):1946-1952.

55. Abiru S, Migita K, Maeda Y, et al. Serum cytokine and soluble cytokine receptor levels in patients with non-alcoholic steatohepatitis. Liver Int. 2006;26(1):39-45.

56. Yilmaz Y. Review article: non-alcoholic fatty liver disease and osteoporosis - clinical and molecular crosstalk. Aliment Pharmacol Ther. 2012;36(4):345-352.

57. Osta B, Benedetti G, Miossec P. Classical and paradoxical effects of TNF- $\alpha$ on bone homeostasis. Front Immunol. 2014;5:48.

58. Weitzmann MN. Bone and the immune system. Toxicol Pathol. 2017; 45(7):911-924.

59. Indridason OS, Franzson L, Sigurdsson G. Serum osteoprotegerin and its relationship with bone mineral density and markers of bone turnover. Osteoporos Int. 2005;16(4):417-423.

60. Szulc P, Hofbauer LC, Heufelder AE, Roth S, Delmas PD. Osteoprotegerin serum levels in men: correlation with age, estrogen, and testosterone status. J Clin Endocrinol Metab. 2001;86(7):3162-3165.

61. Mezquita-Raya P, de La Higuera M, García DF, et al. The contribution of serum osteoprotegerin to bone mass and vertebral fractures in postmenopausal women. Osteoporos Int. 2005;16(11):1368-1374.

62. Kong Y-Y, Boyle WJ, Penninger JM. Osteoprotegerin ligand: A common link between osteoclastogenesis, lymph node formation and lymphocyte development. Immunol Cell Biol. 1999;77(2):188-193.

63. Yilmaz Y, Yonal O, Kurt R, et al. Serum levels of osteoprotegerin in the spectrum of nonalcoholic fatty liver disease. Scand J Clin Lab Invest. 2010;70(8):541-546.

64. Yang M, Liu Y, Zhou G, et al. Value of serum osteoprotegerin in noninvasive diagnosis of nonalcoholic steatohepatitis. Zhonghua Gan Zang Bing Za Zhi. 2016;24:96-101.

65. Niu Y, Zhang W, Yang Z, et al. Plasma osteoprotegerin levels are inversely associated with nonalcoholic fatty liver disease in patients with type 2 diabetes: A case-control study in China. Metabolism. 2016; 65(4):475-481.

66. Stefan N, Häring H-U. The role of hepatokines in metabolism. Nat Rev Endocrinol. 2013;9(3):144-152.

67. Jh I, Shlipak MG, Brandenburg VM, Ali S, Ketteler M, Whooley MA. Association between human fetuin-A and the metabolic syndrome: data from the Heart and Soul Study. Circulation. 2006;113:1760-1767.
68. Xu Y, Xu M, Bi Y, et al. Serum fetuin-A is correlated with metabolic syndrome in middle-aged and elderly Chinese. Atherosclerosis. 2011; 216(1):180-186.

69. Cui Z, Xuan R, Yang Y. Serum fetuin A level is associated with nonalcoholic fatty liver disease in Chinese population. Oncotarget. 2017; 8(63):107149-107156.

70. Haukeland JW, Dahl TB, Yndestad A, et al. Fetuin A in nonalcoholic fatty liver disease: in vivo and in vitro studies. Eur J Endocrinol. 2012; 166(3):503-510.

71. Sato M, Kamada Y, Takeda Y, et al. Fetuin-A negatively correlates with liver and vascular fibrosis in nonalcoholic fatty liver disease subjects. Liver Int. 2015;35(3):925-935.

72. Brylka L, Jahnen-Dechent W. The role of fetuin-A in physiological and pathological mineralization. Calcif Tissue Int. 2013;93(4): 355-364.

73. Chailurkit L, Kruavit A, Rajatanavin R, Ongphiphadhanakul B. The relationship of fetuin-A and lactoferrin with bone mass in elderly women. Osteoporos Int. 2011;22(7):2159-2164.

74. Özkan E, Özkan H, Bilgiç S, et al. Serum fetuin-A levels in postmenopausal women with osteoporosis. Turk J Med Sci. 2014;44:985-988.

75. Gursoy G, Acar Y, Alagoz S. Osteopontin: A multifunctional molecule. J Med Sci. 2010;1:55-60.

76. Rittling SR, Matsumoto HN, Mckee MD, et al. Mice lacking osteopontin show normal development and bone structure but display altered osteoclast formation in vitro. J Bone Miner Res. 1998;13(7):1101-1111.

77. Reza S, Shaukat A, Mahmud M, Arain T, Reza U, Waheed F. Evaluation of osteopontin in combination with bone turnover markers for the assessment of osteoporosis in postmenopausal women. $\mathrm{Br} J \mathrm{Med}$ Med Res. 2016;14(6):1-11.

78. Lund SA, Giachelli CM, Scatena M. The role of osteopontin in inflammatory processes. J Cell Commun Signal. 2009;3(3-4):311-322.

79. Chapman J, Miles PD, Ofrecio JM, et al. Osteopontin is required for the early onset of high fat diet-induced insulin resistance in mice. PLoS One. 2010;5(11):e13959.

80. Kiefer FW, Neschen S, Pfau B, et al. Osteopontin deficiency protects against obesity-induced hepatic steatosis and attenuates glucose production in mice. Diabetologia. 2011;54(8):2132-2142.

81. Rosen CJ, Bouxsein ML. Mechanisms of disease: is osteoporosis the obesity of bone? Nat Clin Pract Rheumatol. 2006;2(1):35-43.

82. Oldberg A, Franzen A, Heinegard D. Cloning and sequence analysis of rat bone sialoprotein (osteopontin) cDNA reveals an ArgGly-Asp cell-binding sequence. Proc Natl Acad Sci. 1986;83(23): 8819-8823.

83. Nomiyama T, Perez-Tilve D, Ogawa D, et al. Osteopontin mediates obesity-induced adipose tissue macrophage infiltration and insulin resistance in mice. J Clin Invest. 2007;117(10):2877-2888.

84. Kwon H-J, Won Y-S, Yoon W-K, Nam K-H, Kim D-Y, Kim H-C. The role of osteopontin in d-galactosamine-induced liver injury in genetically obese mice. Toxicol Appl Pharmacol. 2010;242(3):344-351.

85. Sahai A, Malladi P, Melin-Aldana H, Green RM, Whitington PF. Upregulation of osteopontin expression is involved in the development of nonalcoholic steatohepatitis in a dietary murine model. Am J Physiol Gastrointest Liver Physiol. 2004;287(1):G264-G273.

86. Sahai A, Malladi P, Pan X, et al. Obese and diabetic $d b / d b$ mice develop marked liver fibrosis in a model of nonalcoholic steatohepatitis: role of short-form leptin receptors and osteopontin. Am J Physiol Gastrointest Liver Physiol. 2004;287(5):G1035-G1043.

87. Chiang T-I, Chang I-C, Lee H-S, Lee H, Huang C-H, Cheng Y-W. Osteopontin regulates anabolic effect in human menopausal osteoporosis with intermittent parathyroid hormone treatment. Osteoporos Int 2011;22(2):577-585.

88. Ferron M, Mckee MD, Levine RL, Ducy P, Karsenty G. Intermittent injections of osteocalcin improve glucose metabolism and prevent type 2 diabetes in mice. Bone. 2012;50(2):568-575.

89. Du J, Zhang M, Lu J, et al. Osteocalcin improves nonalcoholic fatty liver disease in mice through activation of Nrf2 and inhibition of JNK Endocrine. 2016;53(3):701-709. 
90. Saleem U, Mosley TH, Kullo IJ. Serum osteocalcin is associated with measures of insulin resistance, adipokine levels, and the presence of metabolic syndrome. Arterioscler Thromb Vasc Biol. 2010; 30(7):1474-1478.

91. Yeap BB, Chubb SAP, Flicker L, et al. Reduced serum total osteocalcin is associated with metabolic syndrome in older men via waist circumference, hyperglycemia, and triglyceride levels. Eur J Endocrinol. 2010;163(2):265-272.

92. Hwang Y-C, Jeong I-K, Ahn K-J, Chung H-Y. Circulating osteocalcin level is associated with improved glucose tolerance, insulin secretion and sensitivity independent of the plasma adiponectin level. Osteoporos Int. 2012;23(4):1337-1342.

93. Zhou B, Li H, Xu L, Zang W, Wu S, Sun H. Osteocalcin reverses endoplasmic reticulum stress and improves impaired insulin sensitivity secondary to diet-induced obesity through nuclear factor- $\kappa \mathrm{B}$ signaling pathway. Endocrinology. 2013;154(3):1055-1068.

94. Yilmaz Y, Kurt R, Eren F, Imeryuz N. Serum osteocalcin levels in patients with nonalcoholic fatty liver disease: Association with ballooning degeneration. Scand J Clin Lab Invest. 2011;71(8):631-636.

95. Amin S, El Amrousy D, Elrifaey S, Gamal R, Hodeib H. Serum osteocalcin levels in children with nonalcoholic fatty liver disease. J Pediatr Gastroenterol Nutr. 2018;66(1):117-121.

96. Luo Y-Qi, Ma X-Jing, Hao Y-Ping, et al. Inverse relationship between serum osteocalcin levels and nonalcoholic fatty liver disease in postmenopausal Chinese women with normal blood glucose levels. Acta Pharmacol Sin. 2015;36(12):1497-1502.

97. Yang HJ, Shim SG, Ma BO, Kwak JY. Association of nonalcoholic fatty liver disease with bone mineral density and serum osteocalcin levels in Korean men. Eur J Gastroenterol Hepatol. 2016;28(3): 338-344.

98. Boutari C, Perakakis N, Mantzoros CS. Association of adipokines with development and progression of nonalcoholic fatty liver disease. Endocrinol Metab. 2018;33(1):33-43.

99. Ebihara K, Kusakabe T, Hirata M, et al. Efficacy and safety of leptinreplacement therapy and possible mechanisms of leptin actions in patients with generalized lipodystrophy. J Clin Endocrinol Metab. 2007;92(2):532-541.

100. Xu A, Wang Y, Keshaw H, Xu LY, Lam KS, Cooper GJ. The fatderived hormone adiponectin alleviates alcoholic and nonalcoholic fatty liver diseases in mice. J Clin Invest. 2003;112(1):91-100.

101. Mirza S, Qu HQ, Li Q, et al. Adiponectin/leptin ratio and metabolic syndrome in a Mexican American population. Clin Invest Med. 2011;34(5):290.

102. Suh HS, Hwang IC, Lee KS, Kim KK. Relationships between serum osteocalcin, leptin and the effect of weight loss by pharmacological treatment in healthy, nonsmoking Korean obese adults. Clin Chim Acta. 2013;418:17-21.

103. Oshima K, Nampei A, Matsuda M, et al. Adiponectin increases bone mass by suppressing osteoclast and activating osteoblast. Biochem Biophys Res Commun. 2005;331(2):520-526.

104. Kanazawa I, Yamaguchi T, Yano S, Yamauchi M, Yamamoto M, Sugimoto T. Adiponectin and AMP kinase activator stimulate proliferation, differentiation, and mineralization of osteoblastic MC3T3-E1 cells. BMC Cell Biol. 2007;8(1):51.

105. Yadav VK, Oury F, Suda N, et al. A serotonin-dependent mechanism explains the leptin regulation of bone mass, appetite, and energy expenditure. Cell. 2009;138(5):976-989.

106. Confavreux CB. Bone: from a reservoir of minerals to a regulator of energy metabolism. Kidney Int. 2011;79(Suppl 121):S14-S19.

107. Musso G, Paschetta E, Gambino R, Cassader M, Molinaro F. Interactions among bone, liver, and adipose tissue predisposing to diabesity and fatty liver. Trends Mol Med. 2013;19(9):522-535.

108. Zeadin MG, Butcher MK, Shaughnessy SG, Werstuck GH. Leptin promotes osteoblast differentiation and mineralization of primary cultures of vascular smooth muscle cells by inhibiting glycogen synthase kinase (GSK)-3ß. Biochem Biophys Res Commun. 2012;425(4): 924-930.
109. Demambro VE, Maile L, Wai C, et al. Insulin-like growth factorbinding protein-2 is required for osteoclast differentiation. J Bone Miner Res. 2012;27(2):390-400.

110. Williams GA, Wang Y, Callon KE, et al. In vitro and in vivo effects of adiponectin on bone. Endocrinology. 2009;150(8):3603-3610.

111. Berner HS, Lyngstadaas SP, Spahr A, et al. Adiponectin and its receptors are expressed in bone-forming cells. Bone. 2004;35(4): 842-849.

112. Mitsui Y, Gotoh M, Fukushima N, et al. Hyperadiponectinemia enhances bone formation in mice. BMC Musculoskelet Disord. 2011;12:18.

113. Tu Q, Zhang J, Dong LQ, et al. Adiponectin inhibits osteoclastogenesis and bone resorption via APPL1-mediated suppression of Akt1. J Biol Chem. 2011;286(14):12542-12553.

114. Wu N, Wang QP, Li H, Wu XP, Sun ZQ, Luo XH. Relationships between serum adiponectin, leptin concentrations and bone mineral density, and bone biochemical markers in Chinese women. Clin Chim Acta. 2010;411(9-10):771-775.

115. Fuggle NR, Westbury LD, Syddall HE, et al. Relationships between markers of inflammation and bone density: findings from the Hertfordshire Cohort Study. Osteoporos Int. 2018;29(7):1581-1589.

116. Tenta R, Kontogianni MD, Yiannakouris N. Association between circulating levels of adiponectin and indices of bone mass and bone metabolism in middle-aged post-menopausal women. J Endocrinol Invest. 2012;35(3):306-311.

117. Thrailkill KM, Lumpkin CK, Bunn RC, Kemp SF, Fowlkes JL. Is insulin an anabolic agent in bone? Dissecting the diabetic bone for clues. Am J Physiol Endocrinol Metab. 2005;289(5):E735-E745.

118. Laurent MR, Cook MJ, Gielen E, et al. Lower bone turnover and relative bone deficits in men with metabolic syndrome: a matter of insulin sensitivity? The European Male Ageing Study. Osteoporos Int. 2016;27(11):3227-3237.

119. Riddle RC, Clemens TL. Insulin, osteoblasts, and energy metabolism: why bone counts calories. J Clin Invest. 2014;124(4):1465-1467.

120. Wei J, Ferron M, Clarke CJ, et al. Bone-specific insulin resistance disrupts whole-body glucose homeostasis via decreased osteocalcin activation. J Clin Invest. 2014;124(4):1781-1793.

121. Srikanthan P, Crandall CJ, Miller-Martinez D, et al. Insulin resistance and bone strength: findings from the study of midlife in the United States. J Bone Miner Res. 2014;29(4):796-803.

122. Xu J, Lombardi G, Jiao W, Banfi G. Effects of exercise on bone status in female subjects, from young girls to postmenopausal women: an overview of systematic reviews and meta-analyses. Sports Med. 2016;46(8):1165-1182.

123. Johnson NA, George J. Fitness versus fatness: moving beyond weight loss in nonalcoholic fatty liver disease. Hepatology. 2010; 52(1):370-380.

124. Zhang HJ, Zhang XF, Ma ZM, et al. Irisin is inversely associated with intrahepatic triglyceride contents in obese adults. $J$ Hepatol. 2013;59(3):557-562.

125. Berzigotti A, Saran U, Dufour JF. Physical activity and liver diseases. Hepatology. 2016;63(3):1026-1040.

126. Arias-Loste MT, Ranchal I, Romero-Gómez M, Crespo J, Irisin CJ. Irisin, a link among fatty liver disease, physical inactivity and insulin resistance. Int J Mol Sci. 2014;15(12):23163-23178.

127. di Iorgi N, Mittelman SD, Gilsanz V. Differential effect of marrow adiposity and visceral and subcutaneous fat on cardiovascular risk in young, healthy adults. Int J Obes. 2008;32(12):1854-1860.

128. Baum T, Yap SP, Karampinos DC, et al. Does vertebral bone marrow fat content correlate with abdominal adipose tissue, lumbar spine bone mineral density, and blood biomarkers in women with type 2 diabetes mellitus? J Magn Reson Imaging. 2012;35(1):117-124.

129. Scheller EL, Rosen CJ. What's the matter with MAT? Marrow adipose tissue, metabolism, and skeletal health. Ann N Y Acad Sci. 2014; 1311:14-30.

130. Fazeli PK, Horowitz MC, Macdougald OA, et al. Marrow fat and bonenew perspectives. J Clin Endocrinol Metab. 2013;98(3):935-945. 
131. Lin H, Grosschedl R. Failure of B-cell differentiation in mice lacking the transcription factor EBF. Nature. 1995;376(6537):263-267.

132. Pongubala JM, Northrup DL, Lancki DW, et al. Transcription factor EBF restricts alternative lineage options and promotes B cell fate commitment independently of Pax5. Nat Immunol. 2008;9(2):203-215.

133. Hesslein DG, Fretz JA, Xi Y, et al. Ebf1-dependent control of the osteoblast and adipocyte lineages. Bone. 2009;44(4):537-546.

134. Fazeli PK, Bredella MA, Misra M, et al. Preadipocyte factor- 1 is associated with marrow adiposity and bone mineral density in women with anorexia nervosa. J Clin Endocrinol Metab. 2010;95(1):407-413.

135. Kuro-OM. Klotho as a regulator of fibroblast growth factor signaling and phosphate/calcium metabolism. Curr Opin Nephrol Hypertens. 2006;15(4):437-441.

136. Hsieh CC, Kuro-OM, Rosenblatt KP, Brobey R, Papaconstantinou J. The ASK1-Signalosome regulates $\mathrm{p} 38$ MAPK activity in response to levels of endogenous oxidative stress in the Klotho mouse models of aging. Aging. 2010;2(9):597-611.

137. Ben-Dov IZ, Galitzer H, Lavi-MoshayoffV, etal. The parathyroid is a target organ for FGF23 in rats. J Clin Invest. 2007;117(12):4003-4008.

138. Somm E, Henry H, Bruce SJ, et al. $\beta$-Klotho deficiency protects against obesity through a crosstalk between liver, microbiota, and brown adipose tissue. JCI Insight. 2017;2(8):e91809.

139. Kurosu H, Kuro-OM. The Klotho gene family as a regulator of endocrine fibroblast growth factors. Mol Cell Endocrinol. 2009;299(1): $72-78$.

140. Dominguez LJ, Barbagallo M. The biology of the metabolic syndrome and aging. Curr Opin Clin Nutr Metab Care. 2016;19(1):5-11.
141. Hsu YH, Venners SA, Terwedow HA, et al. Relation of body composition, fat mass, and serum lipids to osteoporotic fractures and bone mineral density in Chinese men and women. Am J Clin Nutr. 2006; 83(1):146-154.

142. Hwang DK, Choi HJ. The relationship between low bone mass and metabolic syndrome in Korean women. Osteoporos Int. 2010;21(3): 425-431.

143. Madan K, Bhardwaj P, Thareja S, Gupta SD, Saraya A. Oxidant stress and antioxidant status among patients with nonalcoholic fatty liver disease (NAFLD). J Clin Gastroenterol. 2006;40(10):930-935.

144. Li M, Xu Y, Xu M, et al. Association between nonalcoholic fatty liver disease (NAFLD) and osteoporotic fracture in middle-aged and elderly Chinese. J Clin Endocrinol Metab. 2012;97(6):2033-2038.

145. Collier J. Bone disorders in chronic liver disease. Hepatology. 2007;46(4):1271-1278.

146. Solaymani-Dodaran M, Card TR, Aithal GP, West J. Fracture risk in people with primary biliary cirrhosis: a population-based cohort study. Gastroenterology. 2006;131(6):1752-1757.

147. Menon KV, Angulo P, Weston S, Dickson ER, Lindor KD. Bone disease in primary biliary cirrhosis: independent indicators and rate of progression. J Hepatol. 2001;35(3):316-323.

148. Wang D, Liu N, Gao Y, Li P, Tian M. Association between metabolic syndrome and osteoporotic fracture in middle-aged and elderly Chinese peoples. Cell Biochem Biophys. 2014;70(2):1297-1303.

149. Qin L, Yang Z, Zhang W, et al. Metabolic syndrome and osteoporotic fracture: a population-based study in China. BMC Endocr Disord. 2016;16(1):27-30.
Clinical Interventions in Aging

\section{Publish your work in this journal}

Clinical Interventions in Aging is an international, peer-reviewed journal focusing on evidence-based reports on the value or lack thereof of treatments intended to prevent or delay the onset of maladaptive correlates of aging in human beings. This journal is indexed on PubMed Central, MedLine,

\section{Dovepress}

CAS, Scopus and the Elsevier Bibliographic databases. The manuscript management system is completely online and includes a very quick and fair peer-review system, which is all easy to use. Visit http://www.dovepress. com/testimonials.php to read real quotes from published authors. 